PROCEEDINGS OF THE

AMERICAN MATHEMATICAL SOCIETY

Volume 126, Number 8, August 1998, Pages 2291-2297

S 0002-9939(98)04325-1

\title{
SPECTRAL PROPERTIES OF THE OPERATOR OF RIESZ POTENTIAL TYPE
}

\author{
MILUTIN R. DOSTANIĆ
}

(Communicated by Palle E. T. Jorgensen)

\begin{abstract}
For the operator of the Riesz potential type we estimate the second term in the asymptotic behavior of the spectrum and find its "regularized" trace.
\end{abstract}

\section{INTRODUCTION AND NOTATION}

For some classes of integral operators there are methods of finding the first term in the asymptotic of the singular values or eigenvalues (see e.g. [2], [3]). However, finding higher terms is rather difficult and can be realized only in some situations, and depends essentially on the structure of the kernel of an operator. In this paper, we consider the operator of Riesz potential type $A: L^{2}(-1,1) \rightarrow L^{2}(-1,1)$ defined by

$$
A f(x)=\int_{-1}^{1}|x-y|^{\alpha-1} f(y) d y, \quad 0<\alpha<1 .
$$

It occurs in the theory of Liouville fractional integrals (see [10]). It is well known (see e.g. [8]) that if $0<\alpha<1$, then operator $A$ is positive and compact. Denote by $\lambda_{1}(A), \lambda_{2}(A), \ldots$ the eigenvalues of $A$ arranged in decreasing order, according to their multiplicity.

Also it is known [2], [3], [6], [9] that

$$
\lim _{n \rightarrow \infty} n^{\alpha} \lambda_{n}(A)=c(\alpha)
$$

holds, where

$$
c(\alpha)=2 \Gamma(\alpha) \cos \frac{\alpha \pi}{2}\left(\frac{2}{\pi}\right)^{\alpha} .
$$

In what follows we denote by $\int_{a}^{b} L(x, y) \cdot d y$ the integral operator acting on $L^{2}(a, b)$, whose kernel is $L(x, y)$.

For a compact operator $T$ we denote by $s_{n}(T)$ the $n$-th eigenvalue of the operator $|T|=\left(T^{*} T\right)^{\frac{1}{2}}$, i.e. $s_{n}(T)=\lambda_{n}(|T|)$.

Received by the editors January 7, 1997

1991 Mathematics Subject Classification. Primary 47B10.

Key words and phrases. Singular values, eigenvalues, regularized trace.

(C)1998 American Mathematical Society 
By $c_{p}$ we denote the set of the compact operators $T$ such that

$$
|T|_{p}=\left(\sum_{k=1}^{\infty} s_{k}^{p}(T)\right)^{\frac{1}{p}}<\infty .
$$

In particular $c_{1}$ is the set of nuclear operators. From the asymptotic of the eigenvalues of $A$ it follows that $A$ is not nuclear.

\section{RESUlt}

Theorem. a) For every $0<r<1$ the following asymptotic formula holds:

$$
\lambda_{n}(A)=\frac{c(\alpha)}{n^{\alpha}}\left(1+o\left(\frac{1}{n^{r}}\right)\right) \quad\left(c(\alpha)=2 \Gamma(\alpha) \cos \frac{\alpha \pi}{2}\left(\frac{2}{\pi}\right)^{\alpha}\right) .
$$

b) The series $\sum_{n=1}^{\infty}\left(\lambda_{n}(A)-\frac{c(\alpha)}{n^{\alpha}}\right)$ converges and

$$
\sum_{n=1}^{\infty}\left(\lambda_{n}(A)-\frac{c(\alpha)}{n^{\alpha}}\right)=-4^{\alpha} \zeta(1-\alpha)
$$

holds, where $\zeta$ is the Reimann zeta function.

Proof of a). For $0<\alpha<1$ define the function

$$
H(x, y)=\sum_{n=-\infty}^{\infty}\left(|x-y+4 n|^{\alpha-1}-|x+y+4 n+2|^{\alpha-1}\right)
$$

and the operator $B: L^{2}(-1,1) \rightarrow L^{2}(-1,1)$,

$$
B f(x)=\int_{-1}^{1} H(x, y) f(y) d y \text {. }
$$

Let $\varphi_{n}(x)=\sin \frac{n \pi(1+x)}{2}, n=1,2,3, \ldots$, and let

$$
K(\xi)=\int_{-\infty}^{\infty} e^{i t \xi}|t|^{\alpha-1} d t \quad\left(=2 \Gamma(\alpha) \cos \frac{\alpha \pi}{2}|\xi|^{-\alpha} \mid\right) .
$$

Observe that $\left\{\varphi_{n}\right\}_{n=1}^{\infty}$ is an orthonormal basis of $L^{2}(-1,1)$. By direct computation one finds that

$$
\int_{-1}^{1} H(x, y) \varphi_{n}(y) d y=K\left(\frac{n \pi}{2}\right) \varphi_{n}(x) .
$$

Therefore, the operator $B$ is selfadjoint and positive and

$$
\lambda_{n}(B)=\frac{c(\alpha)}{n^{\alpha}} .
$$

From (1) we obtain

$$
\begin{aligned}
|x-y|^{\alpha-1} & =H(x, y)+|x+y+2|^{\alpha-1}+|x+y-2|^{\alpha-1} \\
& +\left(-|x-y-4|^{\alpha-1}-\sum_{n \neq 0,-1}\left(|x-y+4 n|^{\alpha-1}-|x+y+4 n+2|^{\alpha-1}\right)\right) .
\end{aligned}
$$


By the result of Laptev [7], we obtain

$$
s_{n}\left(\int_{-1}^{1}|x+y \pm 2|^{\alpha-1} \cdot d y\right)=O\left(e^{-c_{0}^{\prime} \sqrt{n}}\right)
$$

where $c_{0}^{\prime}>0$ is independent of $n$. On the other hand, by the Birman-Solomjak theorem [2], we have

$$
s_{n}(W)=O\left(e^{-c_{0}^{\prime \prime} n}\right)
$$

where $W$ denotes the operator with the kernel

$$
-|x-y-4|^{\alpha-1}-\sum_{n \neq 0,-1}\left(|x-y+4 n|^{\alpha-1}-|x+y+4 n+2|^{\alpha-1}\right),
$$

and $c_{0}^{\prime \prime}>0$ is independent of $n$.

Hence, by the properties of the singular values of the sum of two operators [5], from (2) it follows that

$$
A=B+R
$$

where

$$
s_{n}(R) \leq c_{1} e^{-c_{0} \sqrt{n}} .
$$

Fix $r, 0<r<1$. Choose $\theta$ so that $r<\theta<1$ and let

$$
\begin{aligned}
& k=k(n)=\left[n^{\theta}\right]-1, \\
& m=m(n)=\left[n^{1-\theta}\right] .
\end{aligned}
$$

Since every natural number $n$ can be represented as $n=(k+1) m+j, j=0,1, \ldots, k$, we obtain the estimate

$$
0 \leq j=j(n) \leq n^{\theta}+n^{1-\theta} .
$$

From the properties of the singular values of the sum two operators it follows that

$$
s_{(k+1) m+j}(B+R) \leq s_{k m+j}(B)+s_{m+1}(R),
$$

i.e.

$$
s_{n}(B+R)-\frac{c(\alpha)}{n^{\alpha}} \leq s_{k m+j}(B)-\frac{c(\alpha)}{n^{\alpha}}+s_{m+1}(R),
$$

i.e.

$$
s_{n}(A)-\frac{c(\alpha)}{n^{\alpha}} \leq c(\alpha)\left(\frac{1}{(k m+j)^{\alpha}}-\frac{1}{n^{\alpha}}\right)+s_{m+1}(R) .
$$

Hence

(4) $\quad n^{\alpha+r}\left(s_{n}(A)-\frac{c(\alpha)}{n^{\alpha}}\right) \leq c(\alpha) n^{\alpha+r}\left(\frac{1}{(k m+j)^{\alpha}}-\frac{1}{n^{\alpha}}\right)+n^{\alpha+r} s_{m+1}(R)$.

From the definition of the sequences $k(n), m(n)$ and $j(n)$ and from (3) it follows that

$$
\varlimsup_{n \rightarrow \infty} n^{\alpha+r}\left(\frac{1}{(k m+j)^{\alpha}}-\frac{1}{n^{\alpha}}\right)=0
$$

and

$$
\varlimsup_{n \rightarrow \infty} n^{\alpha+r} s_{m+1}(R)=0 .
$$


Hence, by (4)

$$
\varlimsup_{n \rightarrow \infty} n^{\alpha+r}\left(s_{n}(A)-\frac{c(\alpha)}{n^{\alpha}}\right) \leq 0
$$

Similarly, starting from

$$
s_{k m+j}(B+R) \geq s_{(k+1) m+j}(B)-s_{m+1}(R),
$$

we get

$$
\underline{\lim }_{n \rightarrow \infty} n^{\alpha+r}\left(s_{n}(A)-\frac{c(\alpha)}{n^{\alpha}}\right) \geq 0
$$

thus $\lim _{n \rightarrow \infty} n^{\alpha+r}\left(s_{n}(A)-\frac{c(\alpha)}{n^{\alpha}}\right)=0$. Since $A>0$ we have $s_{n}(A)=\lambda_{n}(A)$ and therefore

$$
\lambda_{n}(A)=\frac{c(\alpha)}{n^{\alpha}}\left(1+o\left(\frac{1}{n^{r}}\right)\right) .
$$

Remark 1 . The above method does not make it possible to answer whether the following formula holds:

$$
\lambda_{n}(A)=\frac{c(\alpha)}{n^{\alpha}}\left(1+O\left(\frac{1}{n}\right)\right) .
$$

Proof of b). From a) it follows that

$$
\lambda_{n}(A)=\frac{c(\alpha)}{n^{\alpha}}+o\left(\frac{1}{n^{r+\alpha}}\right),
$$

hence, for $r>1-\alpha$, the series $\sum_{n=1}^{\infty}\left(\lambda_{n}(A)-\frac{c(\alpha)}{n^{\alpha}}\right)$ converges. Moreover for all $k=1,2, \ldots$ the series

$$
\sum_{n=1}^{\infty}\left(\lambda_{n}^{k}(A)-\left(\frac{c(\alpha)}{n^{\alpha}}\right)^{k}\right)
$$

is convergent. For the proof of b) we need a lemma.

Lemma. If $C$ and $D$ are positive, compact operators on some complex Hilbert space such that $|C-D|^{\operatorname{Re} z} \in c_{1}(0<\operatorname{Re} z<1)$, then $C^{z}-D^{z} \in c_{1}$ and

$$
\left|C^{z}-D^{z}\right|_{1} \leq \frac{|\sin \pi z|}{\sin (\pi \cdot \operatorname{Re} z)}|| C-\left.\left.D\right|^{\operatorname{Re} z}\right|_{1} .
$$

Proof. If $|C-D|^{\operatorname{Re} z} \in c_{1}$ for some $z, 0 \leq \operatorname{Re} z<1$, then $|C-D| \in c_{1}$ and hence $C(I+t C)^{-1}-D(I+t D)^{-1} \in c_{1}$ for all $t>0$ ( $I$ is the identity operator). Since, for $0<\operatorname{Re} z<1$,

$$
C^{z}-D^{z}=\frac{\sin \pi z}{\pi} \int_{0}^{\infty} t^{-z}\left(C(I+t C)^{-1}-D(I+t D)^{-1}\right) d t
$$


and since the function $s \mapsto \frac{s}{1+t s}$ is operator monotone, according to a result of T. Ando [1], we have

$$
\begin{aligned}
\left|C^{z}-D^{z}\right|_{1} & \leq \frac{|\sin \pi z|}{\pi} \int_{0}^{\infty} t^{-\operatorname{Re} z}\left|C(I+t C)^{-1}-D(I+t D)^{-1}\right|_{1} d t \\
& \leq \frac{|\sin \pi z|}{\pi} \int_{0}^{\infty} t^{-\operatorname{Re} z}|| C-D\left|(I+t|C-D|)^{-1}\right|_{1} d t \\
& =\frac{|\sin \pi z|}{\pi} \sum_{k=1}^{\infty} s_{k}^{\operatorname{Re} z}(C-D) \cdot \int_{0}^{\infty} \frac{t^{-\operatorname{Re} z}}{1+t} d t \\
& =\frac{|\sin \pi z|}{\sin (\pi \operatorname{Re} z)}|| C-\left.\left.D\right|^{\operatorname{Re} z}\right|_{1} .
\end{aligned}
$$

Applying the lemma to the operators $C=A, D=B$ we obtain from (3) that $|A-B|^{\operatorname{Re} z} \in c_{1}$ for every $z, 0<\operatorname{Re} z<1$, and hence $A^{z}-B^{z} \in c_{1}$. If $0<\operatorname{Re} z<2$, then applying the lemma to $C=A^{2}, D=B^{2}$ we obtain $\left(A^{2}\right)^{\frac{z}{2}}-\left(B^{2}\right)^{\frac{z}{2}} \in c_{1}$, etc. Therefore $A^{z}-B^{z} \in c_{1}$ for all $z$ with $\operatorname{Re} z>0$. In a similar way one shows that the function

$$
z \mapsto \operatorname{tr}\left(A^{z}-B^{z}\right)
$$

is analytic for $\operatorname{Re} z>0$.

Consider the function

$$
\Psi(z)=\sum_{n=1}^{\infty}\left(\lambda_{n}^{z}(A)-\left(\frac{c(\alpha)}{n^{\alpha}}\right)^{z}\right) .
$$

From the asymptotic of $\lambda_{n}(A)$ it follows that $\Psi(z)$ is analytic for $\operatorname{Re} z>0$. If $\operatorname{Re} z>\frac{1}{\alpha}$, then both operators $A^{z}$ and $B^{z}$ are nuclear and hence

$$
\operatorname{tr}\left(A^{z}-B^{z}\right)=\operatorname{tr} A^{z}-\operatorname{tr} B^{z}=\Psi(z) .
$$

Hence $\Psi(z)=\operatorname{tr}\left(A^{z}-B^{z}\right)$ for $\operatorname{Re} z>\frac{1}{\alpha}$. It follows that $\Psi(z)=\operatorname{tr}\left(A^{z}-B^{z}\right)$ for all $z, \operatorname{Re} z>0$, and hence

$$
\sum_{n=1}^{\infty}\left(\lambda_{n}^{k}(A)-\left(\frac{c(\alpha)}{n^{\alpha}}\right)^{k}\right)=\operatorname{tr}\left(A^{k}-B^{k}\right), \quad k=1,2, \ldots
$$

It is especially simple to find the "first regularized" trace of $A$ :

$$
\sum_{n=1}^{\infty}\left(\lambda_{n}(A)-\frac{c(\alpha)}{n^{\alpha}}\right)=\operatorname{tr}(A-B)=\operatorname{tr} R
$$

The integral operator, on $L^{2}(-1,1)$, with the continuous kernel

$$
-|x-y-4|^{\alpha-1}-\sum_{n \neq 0,-1}\left(|x-y+4 n|^{\alpha-1}-|x+y+4 n+2|^{\alpha-1}\right)
$$

is nuclear and its trace is equal [5] to

$$
\int_{-1}^{1}\left[-4^{\alpha-1}-\sum_{n \neq 0,-1}\left(|4 n|^{\alpha-1}-|2 x+4 n+2|^{\alpha-1}\right)\right] d x .
$$


The operators $\int_{-1}^{1}|x+y+2|^{\alpha-1} \cdot d y$ and $\int_{-1}^{1}|x+y-2|^{\alpha-1} \cdot d y$ are nuclear and, by [4], Theorem 3.1, it follows that

$$
\begin{aligned}
& \operatorname{tr} \int_{-1}^{1}|x+y+2|^{\alpha-1} \cdot d y=\int_{-1}^{1}|2 x+2|^{\alpha-1} d x=\frac{4^{\alpha}}{2 \alpha} \\
& \operatorname{tr} \int_{-1}^{1}|x+y-2|^{\alpha-1} \cdot d y=\int_{-1}^{1}|2 x+2|^{\alpha-1} d x=\frac{4^{\alpha}}{2 \alpha} .
\end{aligned}
$$

Hence

$$
\operatorname{tr} R=2 \cdot \frac{1}{2 \alpha} \cdot 4^{\alpha}+\int_{-1}^{1}\left[-4^{\alpha-1}-\sum_{n \neq 0,-1}\left(|4 n|^{\alpha-1}-|2 x+4 n+2|^{\alpha-1}\right)\right] d x .
$$

After simplification we get

$$
\operatorname{tr} R=-4^{\alpha} \zeta(1-\alpha) .
$$

This completes the proof of the theorem.

Remark 2. A similar result can be obtained for the operator $L: L^{2}(-1,1) \rightarrow$ $L^{2}(-1,1)$ defined by

$$
L f(x)=-\frac{1}{\pi} \int_{-1}^{1} \ln |x-y| f(y) d y .
$$

It is known [8] that $L>0$ and $\lambda_{n}(L)=\frac{2}{n \pi}\left(1+O\left(\frac{\sqrt{\ln n}}{n^{\frac{1}{5}}}\right)\right)$. Starting from the function $k(x)=\frac{1}{\pi} K_{0}(|x|)\left(K_{0}\right.$ is the McDonald function), one can prove the formula

$$
\begin{aligned}
& \sum_{n=1}^{\infty}\left(\lambda_{n}(L)-\frac{1}{\sqrt{1+\left(\frac{n \pi}{2}\right)^{2}}}\right) \\
& \quad=-\frac{2}{\pi}(C+\ln 2)-\frac{2 \sqrt{2}}{\pi} \int_{0}^{\infty} t^{-\frac{1}{2}}\left(1+\frac{t}{2}\right)^{-\frac{1}{2}} \frac{d t}{e^{4(1+t)}-1}
\end{aligned}
$$

where $C$ is the Euler constant.

Remark 3. By the same method one can find the "regularized" trace of the convolution operator with kernel of the form

$$
k(x)=|x|^{\alpha-1} L\left(\frac{1}{|x|}\right) \quad(0<\alpha<1)
$$

where $L$ is a smooth function such that

$$
x \mapsto \frac{x L^{\prime}(x)}{L(x)}
$$

decreases for $x$ large enough and

$$
\lim _{x \rightarrow \infty} \frac{x L^{\prime}(x)}{L(x)}=0 .
$$




\section{REFERENCES}

[1] T. Ando, Comparison of norms $\|f(A)-f(B)\|$ and $\|f(|A-B|)\|$, Math. Zeit. 197 (1988), 403-408. MR 90a:47021

[2] M. Š. Birman and M. Z. Solomjak, Estimates of singular values of the integral operators, Uspekhi Mat. Nauk. 32, No. 1 (193) (1977), 17-84. MR 55:11104

[3] M. Š. Birman and M. Z. Solomjak, Asymptotic behaviour of the spectrum of weakly polar integral operators, Izv. Akad. Nauk SSSR Ser. Mat. 34 (1970), No. 5. MR 43:5359

[4] C. Brislawn, Kernels of trace class operators, Proc. Amer. Math. Soc. 104 (1988), 1180-1190. MR 89d: 47059

[5] I. C. Gohberg and M. G. Krein, "Introduction to the theory of linear nonselfadjoint operators", Translation of the math. monographs, Vol. 18, Amer. Math. Soc., Providence, R.I. 1969. MR 39:7447

[6] M. Kac, Distribution of eigenvalues of certain integral operators, Michigan Math. J. 3 (195556), 141-148. MR 19:70j

[7] A. Laptev, The spectral asymptotic of a class of integral operators, Mat. Zametki 16, No. 5, (1974), 741-750. MR 51:8894

[8] J. B. Rid, Asymptotic behaviour of eigenvalues of certain integral equations, Proceedings of the Edinburgh Mat. Soc. 22 (1979), 137-144.

[9] M. Rosenblatt, Some results on the asymptotic behavior of eigenvalues for a class of integral equations with translation kernels, J. Math. Mech. 12 (1963), 619-628. MR 27:547

[10] S. G. Samko, A. A. Kilbas, and O. I. Maricev, Fractional integrals and derivations and some applications, Minsk, 1987. MR 89a:26009

Matematicki Fakultet, Studentski trg16, 11000 Beograd, Yugoslavia 